\title{
34
}

\section{Discussion Group 2 - Policies, strategies $\&$ initiatives of ICT in education}

\author{
Chair: Sam Gumbo (Zimbabwe) \\ Rapporteur: Pieter Hogenbirk (The Netherlands) \\ Participants: Walter Chakuzira (Zimbabwe), Peter Hubwieser (Germany), Geoffrey \\ Kiangi (Namibia), Olivia Magaya (Zimbabwe), Marshall Mlambo (Zimbabwe), \\ Joanna Marivara (Zimbabwe), Rudo Mataranyika (Zimbabwe), Simon Mphisa \\ (Zimbabwe), Hajime Ohiwa (Zimbabwe), Charles Nherera (Zimbabwe), Sindre \\ Røsvik (Norway), Simon Satumba (Zimbabwe), Obert Sifile (Zimbabwe), Enock \\ Tinofirei (Zimbabwe), Lorraine Tafira (Zimbabwe), Marsha Williams (USA)
}

\section{INTRODUCTION}

Discussing the theme of policies, strategies and successful initiatives for Information and Communication Technology (ICT), one has to consider first the objectives. From these objectives, policies can be derived to meet the goals. Policies still are general statements, which have to be worked out in elements of strategy. In the last stage of elaborating on this theme the elements of strategy need to be made operational into activities and actions which might be the most successful to achieve the originally formulated objectives. We actually decided not to split up the discussion into the three levels of education - primary, secondary and tertiary education - in order to get a framework that could apply to all of these levels. So it was agreed to structure the discussion in the following four stages:

- definition of the targets, needs, objectives or goals one wants to achieve with respect to ICT in education - (the 'why');

- development from this of various aspects of policy making (the 'what');

- development of the strategy elements fitting to the policies (the 'how');

- implementation of the strategies by activities that might be most successful.

The framework we used made the discussion very clear and fruitful. Nevertheless we should make one remark at the beginning. We should not bother too much about whether something is a policy, an objective, a strategy or a down-to-earth activity. It is very important that policy makers are aware of the choices to be made in strategies and easy-to-implement activities. At the same time the educational community should recognize the fact that innovations and changes have a need for support and approval of the politicians and the industry. By understanding the

\footnotetext{
Capacity Building for IT in Education in Developing Countries

G. Marshall \& M. Ruohonen (Eds.)

() 1998 IFIP. Published by Chapman \& Hall
} 
mutual interests stakeholders and participants in the process could be involved to a level needed for making the innovation a success.

Objectives could be defined from different perspectives. They could be social, applying to the needs of the society; they could be vocational, aiming at professional development; they could be pedagogical, affecting the actual teaching process; and they could be catalytic, stimulating other innovations and changes to occur. We established the following list of objectives which involve the four categories. All of the objectives apply to the pupils, the teachers, the administrators as well as the teacher trainers - the whole of the educational community.

- You should be able to use the computer as a tool for your personal functioning.

- You should be able to use ICT for communication and information retrieval.

- You should know the potential and implications of ICT.

- You should achieve ICT-abilities for future professional development.

- You should be able to participate actively in the global society, not widening the gap between the 'haves' and 'have nots' any more.

- The use of ICT should catalyze and improve the quality of the learning process.

- There should be more capacity building on ICT-use and research in the local language, culture and national programs.

3

\section{POLICIES}

To achieve these objectives we need to have policies - not only written papers but actual statements that could be translated later into strategies for achieving the policies. We came up with the following policy statements:

- Every child and teacher should have access to the use of ICT. This policy is often referred to as the issue of accessibility.

- Males and females should have equal opportunity in using ICT. This policy is often referred to as the equity issue.

- There should be a development of national curriculum aspects for ICT, shortterm and long-term. These elements cover ICT in separate subjects as well as ICT integrated in other subjects.

- All relevant stakeholders (government, industries, educational community, local communities, parents) should be involved when that is relevant and essential for the strategy.

- There should be an intensive program for teacher training going along with curriculum development and technical development.

- A basic infrastructure of hardware, software and connectivity should be defined and implemented, which enables all kinds of activities and projects to work together. 
- There should be set up a system for the accreditation and certification of the ICT programs as well as for the assessment of ICT objectives in the curriculum.

- There should be a thorough financing programme for achieving the objectives. The plan of actions should be defined in terms of feasible outcomes, depending on the budgets available.

\section{STRATEGIES}

Still the policies are phrased in a rather general way. They have to be translated into strategic actions and ideas to make them work. Note that the strategies could be streamlined along various aspects, e.g. national, regional and school level. We did not do so because the list will not be exhaustive. Yet we can divide the strategies into two categories. At first we state a preliminary idea (A). The first set of strategies (B-I) applies to ideas and actions to be taken for elaborating on the policies. The second category ( $\mathrm{J}-\mathrm{O}$ ) consists of actual activities, in general terms, to be undertaken. This leads to the following list:

A. The strategy should have both top-down and bottom-up elements. This will provide a balance for responsibility and involvement of the relevant participants in the process.

To implement the policies the following actions could be taken:

B. A major effort should be made to influence policy makers and politicians. They are the key persons when it comes to making up the general policy statements and the funding of the process.

C. Plans of activities should be tied up firmly to national development programs and perspectives for the necessary governmental support.

D. The involvement of administrators is needed to back up the teachers, the parents and the local communities.

E. Collaboration between public and private sectors should be established by appealing to their mutual responsibilities and abilities.

F. To stimulate such collaboration we should create and enable an environment with incentives for industries and major representatives of the public sector to get involved and take responsibility.

$\mathrm{G}$. The development of educational software is very expensive. So one should try to use existing software when possible and at the same time have some good examples of software that fit the national language(s) and culture.

$\mathrm{H}$. To narrow the gap between developed and developing countries there should be investigated the development of research centers for assembling and manufacturing ICT tools.

I. To be part of the global society and participating members of the world community we should network all schools to public offices, to other schools and to the Internet.

Some strategies can be formulated much more as general activities. In the next phase these could be translated today to start initiatives. We thought of the following list: 
J. We should define a syllabus for ICT as a subject and integrate it (as a catalyst) into other subjects.

$\mathrm{K}$. We have to define and start experimental projects that are provided with all resources needed.

L. We have to start pilot projects in which the main objectives have to be to investigated to see if they are replicable and to see how experiences gained from them could be multiplied.

M. Most important is the development and orientation of the human resources, synchronized with the technical equipment and the curriculum development, and giving people time to change and to adapt to the new situation. So an integrated and step-by-step approach will be necessary. Human resources imply:

- M1. the administrators,

- M2. the teachers,

- M3. the technicians,

- M4. the teacher trainers.

$\mathrm{N}$. Especially in developing countries it is very important to get the local communities involved so they should be mobilized.

$O$. There should be given preferential treatment to disadvantaged schools, e.g. by divising the means in a way they got more than the more privileged schools.

\section{5 \\ SUCCESSFUL ACTIVITIES FOR IMPLEMENTING STRATEGIES}

The strategic elements have to be translated into 'on-the-floor' initiatives. The discussion group brainstormed about activities with a low threshold, strategies which could be started today. Added to each of these activities is a reference to the list of strategies above. The order of these activities is just coincidental.

- Industrial parties should invite politicians to influence their thinking and views (B, D).

- From successful sites there could be organized demonstrations and sessions for training ICT skills for administrators and teachers (C, M1).

- All participants involved have to share responsibilities for project and their outcomes, e.g. by signing a mutual agreement and by all contributing financially $(\mathrm{C}, \mathrm{M})$.

- Professional networks of teachers and technicians - via the Computer Teacher Association (CTA) - should be established (M2, M3).

- It was emphasized that a flourishing ICT Teachers Association should be built.

- Schools should try to get used equipment from industry and from abroad $(\mathrm{N}$, D). National and international organizations have to take the lead for that.

- Stakeholders must organize community outreach (M).

- Schools and training institutes can make use of parents, teachers, assistants, school development associations, computer associations, etc. for giving good examples and helping in capacity building (M). 
- Research projects on assembling and manufacturing computers and software can be initiated (F, G).

- As in the European countries the European drivers licence could be introduced, including keyboard skills (L).

- Software could be developed that fits the local cultural environment and is written in the national language(s) (F).

\section{CONCLUSIONS}

The discussion on the themes in the framework presented above, led us to the following conclusions:

- The implementation of a policy needs an integrated approach where all policy and strategy elements are conducted together. Such an approach must include the availability of infrastructure (hardware and software), the human resources development ('humanware'), the involvement of the (local) community and the implementation of the process (procedures).

- This integrated approach should be made operational in a clear plan of activities that includes a clear financial paragraph. This plan of action should take full advantage of all potential resources.

- The involvement of strategic people, bodies and institutions is fundamental for taking responsibility in both the set-up of the process and the implementation. 